\title{
PROPERTY (FA) OF THE GAUSS-PICARD MODULAR GROUP
}

\author{
JIEYAN WANG and BAOHUA XIE ${ }^{\bowtie}$
}

(Received 12 October 2010)

\begin{abstract}
In this note, we prove that the Gauss-Picard modular group $\mathbf{P U}\left(2,1 ; \Theta_{1}\right)$ has Property (FA). Our result gives a positive answer to a question by Stover ['Property (FA) and lattices in SU(2,1)', Internat. J. Algebra Comput. 17 (2007), 1335-1347] for the group PU(2, 1; $\left.\Theta_{1}\right)$.
\end{abstract}

2010 Mathematics subject classification: primary 30F40; secondary 22E40, $20 \mathrm{H} 10$.

Keywords and phrases: Picard modular group, Property (FA).

\section{Introduction}

Whether a group $G$ has Property (FA) is an important question in the study of lattices in semisimple Lie groups. In the study of Property (FA), there is a fundamental theorem due to Serre [6].

THEOREM 1.1. A group $G$ has Property (FA) if and only if:

(1) $G$ is finitely generated;

(2) $G$ does not split as a nontrivial free product with amalgamation;

(3) $G$ does not admit a homomorphism onto $\mathbb{Z}$.

Since the irreducible lattices in $\mathbf{S p}(n, 1)$ for $n \geq 2, \mathbf{F}_{4(-20)}$, and semisimple Lie groups with $\mathbb{R}$-rank at least two always have Property (FA) (see [1]), the remaining interesting cases are the fundamental groups of real and complex hyperbolic manifolds, that is, lattices in $\mathbf{P S O}_{0}(n, 1)$ and $\mathbf{P U}(n, 1)$.

In [5] there are many cocompact Fuchsian groups, that is, lattices in $\operatorname{PSL}(2, \mathbb{R})$, which split as a free product with amalgamation. It is well known that cocompact Fuchsian triangle groups have Property (FA) and the classical modular group $\operatorname{PSL}(2, \mathbb{Z})$ does not have Property $(\mathrm{FA})$, since $\operatorname{PSL}(2, \mathbb{Z})$ is a free product of two finite cyclic groups $\mathbb{Z}_{2}$ and $\mathbb{Z}_{3}$.

Let $\Theta_{d}$ denote the ring of algebra integers in the quadratic number field $\mathbb{Q}(\sqrt{-d})$, where $d$ is a square-free positive integer. In [3] Frohman and Fine proved that the and B. Xie was also supported by Hunan University (No. 531107040021).

(C) 2011 Australian Mathematical Publishing Association Inc. 0004-9727/2011 \$16.00 
Bianchi group PSL $\left(2 ; \Theta_{d}\right)$ splits as a nontrivial free product with amalgamation for $d \neq 3$. But in [6] Serre proved that PSL $\left(2 ; \Theta_{3}\right)$ has Property (FA).

As the complex hyperbolic analogue of Bianchi group $\operatorname{PSL}\left(2 ; \Theta_{d}\right)$, the group $\mathbf{P U}\left(2,1 ; \Theta_{d}\right)$ is called the Picard modular group, which is a subgroup of $\mathbf{P U}(2,1)$ with entries in $\Theta_{d}$.

The study of Property (FA) of Picard modular groups was begun by Stover in [7], where the author proved the following theorem.

Theorem 1.2. $\mathbf{P U}\left(2,1 ; \Theta_{3}\right)$ and $\mathbf{S U}\left(2,1 ; \Theta_{3}\right)$ have Property $(F A)$.

This theorem indicates that there is a connection between certain real and complex hyperbolic lattices. In the same paper [7], Stover asked the following question.

Question 1.3. Does PU(2, $\left.1 ; \Theta_{d}\right)$ or $\mathbf{S U}\left(2,1 ; \Theta_{d}\right)$ have Property (FA) for $d \neq 3$ ?

The aim of this note is to show the following result.

Theorem 1.4. PU(2, $\left.1 ; \Theta_{1}\right)$ and $\mathbf{S U}\left(2,1 ; \Theta_{1}\right)$ have Property $(F A)$.

\section{Preliminaries}

2.1. Complex hyperbolic space. In this subsection, we recall some basic material about complex hyperbolic space. More details can be found in [2, 4].

Let $\mathbb{C}^{2,1}$ denote the three-dimensional complex vector space $\mathbb{C}^{3}$ equipped with the Hermitian form

$$
\langle z, w\rangle=z_{1} \bar{w}_{3}+z_{2} \bar{w}_{2}+z_{3} \bar{w}_{1},
$$

where $z=\left(z_{1}, z_{2}, z_{3}\right)^{t}$ and $w=\left(w_{1}, w_{2}, w_{3}\right)^{t}$. The vector $x^{t}$ stands for the transpose of vector $x$. Consider the subspaces of $\mathbb{C}^{2,1}$ :

$$
\begin{aligned}
& V_{-}=\left\{z \in \mathbb{C}^{2,1} \mid\langle z, z\rangle<0\right\}, \\
& V_{0}=\left\{z \in \mathbb{C}^{2,1}-\{0\} \mid\langle z, z\rangle=0\right\} .
\end{aligned}
$$

Complex hyperbolic space $\mathbf{H}_{\mathbb{C}}^{2}$ is defined to be the complex projective subspace $\mathbb{P}\left(V_{-}\right)$equipped with the Bergman metric, where $\mathbb{P}: \mathbb{C}^{2,1}-\{0\} \rightarrow \mathbb{C} P^{2}$ is the canonical projection onto the complex projective space. We consider the complex hyperbolic space $\mathbf{H}_{\mathbb{C}}^{2}$ as the Siegel domain $\left\{z=\left.\left(z_{1}, z_{2}\right) \in \mathbb{C}^{2}\left|2 \Re\left(z_{1}\right)+\right| z_{2}\right|^{2}<0\right\}$. The boundary of complex hyperbolic space is $\partial \mathbf{H}_{\mathbb{C}}^{2}=\mathbb{P}\left(V_{0}\right)$, which can be identified with the one-point compactification $\overline{\mathfrak{N}}$ of the Heisenberg group $\mathfrak{N}$ by stereographic projection. The point at infinity is $q_{\infty}=(1,0,0)^{t}$.

The group of biholomorphic transformations of complex hyperbolic space $\mathbf{H}_{\mathbb{C}}^{2}$ is $\mathbf{P U}(2,1)$, which is the projectivization of the unitary group $\mathbf{U}(2,1)$ preserving the Hermitian form. If we consider the special unitary group $\mathbf{S U}(2,1)$, it is clear that $\mathbf{S U}(2,1)$ is a threefold cover of $\mathbf{P U}(2,1)$ by the subgroup $\left\{I, \omega I, \omega^{2} I\right\}$, where $I$ stands for the identity matrix and $\omega$ stands for the primitive cube root of unity.

2.2. Property (FA). Let $G$ be a group, and $\Upsilon$ be a tree with an action by $G$. Let $\Upsilon^{G}$ denote the subtree of fixed points of the $G$-action. We say that $G$ has Property (FA) 
if $\Upsilon^{G} \neq \emptyset$ for every tree $\Upsilon$ on which $G$ acts without inversions. Although Theorem 1.1 is fundamental, we have the following two propositions which will be crucial in the proof of Theorem 1.4 in the next section.

Proposition 2.1 [7, Proposition 2.4]. Suppose that $G$ is a finitely presented group and $N \unlhd G$ a normal subgroup such that $N$ and $G / N$ have Property (FA). Then $G$ also has Property (FA).

Proposition 2.2 [7, Proposition 2.5]. Suppose that $G$ is a group with subgroups $A=\left\langle a_{i}\right\rangle$ and $B=\left\langle b_{j}\right\rangle$ with $G=\langle A, B\rangle$ and that $G$ acts on a tree $\Upsilon$. If $\Upsilon^{A}, \Upsilon^{B} \neq \emptyset$ and every $a_{i} b_{j}$ has a fixed point on $\Upsilon$, then $\Upsilon^{G} \neq \emptyset$.

\section{Proof of Theorem 1.4}

In this section we give a proof of Theorem 1.4 which is similar to the proof of Theorem 1.2 in [7].

Let $\mathscr{D}\left(\Theta_{1}\right)$ denote the diagonal subgroup of $\mathbf{S U}\left(2,1 ; \Theta_{1}\right)$ and $\mathscr{N}\left(\Theta_{1}\right)$ denote the subgroup of strictly upper triangular matrices. The Borel subgroup of upper triangular matrices is

$$
\mathscr{B}\left(\Theta_{1}\right)=\mathscr{N}\left(\Theta_{1}\right) \rtimes \mathscr{D}\left(\Theta_{1}\right) .
$$

It is clear that the Borel subgroup of $\mathbf{P U}\left(2,1 ; \Theta_{1}\right)$, which is the projectivization of the Borel subgroup in $\mathbf{S U}\left(2,1 ; \Theta_{1}\right)$, equals the subgroup $\Gamma_{\infty}$, the stabilizer of $q_{\infty}$ in $\mathbf{P U}\left(2,1 ; \Theta_{1}\right)$. The following theorem, proved by Falbel et al. in [2], is crucial in the proof of Theorem 1.4.

TheOrem 3.1. The Gauss-Picard modular group $\mathbf{P U}\left(2,1 ; \Theta_{1}\right)$ has a presentation

$$
\begin{aligned}
& \left\langle I_{0}, Q, T: I_{0}^{2}=Q^{2}=\left(I_{0} Q\right)^{3}=\left(I_{0} T\right)^{12}=\left(I_{0} Q T\right)^{8}=\left[\left(I_{0} T\right)^{3}, T\right]\right. \\
& \quad=[Q, T]=\text { Identity }\rangle .
\end{aligned}
$$

We use the same notation as in [2]. Furthermore, Falbel et al. [2] proved that the Gauss-Picard modular group can be generated by $R, Q, T, I_{0}$ and that the Borel subgroup $\Gamma_{\infty}$ has the presentation

$$
\Gamma_{\infty}=\left\langle R, Q, T: Q^{2}=R^{4}=\left(R^{-1} Q T\right)^{4}=[R, T]=[Q, T]=\text { Identity } .\right.
$$

Proof of Theorem 1.4. It is clear that the groups $\mathbf{S U}\left(2,1 ; \Theta_{1}\right)$ and $\mathbf{P U}\left(2,1 ; \Theta_{1}\right)$ are isomorphic, since there is a unique cube root of unity in $\Theta_{1}$. Hence it is enough to prove that the Gauss-Picard modular group $\mathbf{P U}\left(2,1 ; \Theta_{1}\right)$ has Property (FA).

Firstly, we prove that the Borel subgroup $\Gamma_{\infty}$ has Property (FA). To do this, according to Theorem 1.1 , we need to show that $\Gamma_{\infty}$ cannot map onto $\mathbb{Z}$ and cannot split as a free product with amalgamation. Assume that the Borel subgroup can map onto $\mathbb{Z}$; then we get a contradiction by considering the presentation of the group. Therefore, the Borel subgroup cannot map onto $\mathbb{Z}$.

To show that the Borel subgroup cannot split as a nontrivial product with amalgamation, we consider the short exact sequence

$$
1 \longrightarrow \mathbb{Z} \longrightarrow \Gamma_{\infty} \longrightarrow \Delta \longrightarrow 1,
$$


described in [2, Proposition 2, Section 3]. The subgroup $\Delta \subset \operatorname{Isom}(\mathbb{Z}[i])$ is of index two, and generated by a rotation $\widehat{Q}$ of order two and another rotation $\widehat{R}$ of order four. We also have $(\widehat{Q} \widehat{R})^{4}=1$. According to Proposition 2.2 the group $\Delta$ has Property (FA), so it cannot split as a free product with amalgamation. Now suppose that $\Gamma_{\infty}$ can split as a free nontrivial product with amalgamation. Since the $\mathbb{Z}$ factor is central in $\Gamma_{\infty}$, the subgroup $\mathbb{Z}$ must be contained in the amalgamation subgroup. It follows from the short exact sequence that the group $\Delta$ can split as a nontrivial free product with amalgamation. This is a contradiction. Hence the Borel subgroup has Property (FA).

Finally, we show that the group $\mathbf{P U}\left(2,1 ; \Theta_{1}\right)$ has Property (FA) by applying Proposition 2.2. We know that

$$
\mathbf{P U}\left(2,1 ; \Theta_{1}\right)=\left\langle I_{0}, \Gamma_{\infty}\right\rangle=\left\langle I_{0},\langle R, T, Q\rangle\right\rangle .
$$

Since $\left\langle I_{0}\right\rangle=\mathbf{Z} / 2 \mathbb{Z}$ is a finite group, clearly it has Property (FA). We have shown that $\Gamma_{\infty}$ has Property (FA). Now let us consider an action of $\mathbf{P U}\left(2,1 ; \Theta_{1}\right)$ on a tree $\Upsilon$. We know that $\Upsilon^{\left\langle I_{0}\right\rangle}, \Upsilon^{\langle R, T, Q\rangle} \neq \emptyset$. In order to prove that the products $I_{0} R, I_{0} T$ and $I_{0} Q$ have fixed points on $\Upsilon$, we just need to show that these elements have finite order. This follows from the presentation of $\mathbf{P U}\left(2,1 ; \Theta_{1}\right)$, which is $\left(I_{0} Q\right)^{3}=\left(I_{0} R\right)^{4}=$ $\left(I_{0} T\right)^{12}=$ Identity. So we have shown that $\mathbf{P U}\left(2,1 ; \Theta_{1}\right)$ has Property $(\mathrm{FA})$ and this completes the proof.

\section{Acknowledgement}

We would like to thank the referee for his/her many helpful comments.

\section{References}

[1] P. de la Harpe and A. Valette, 'La propriété (T) de Kazhdan pour les groupes localement compacts (avec un appendice de Marc Burger)', Astérisque 175 (1989).

[2] E. Falbel, G. Francsics and J. R. Parker, 'The geometry of the Gauss-Picard modular group', Math. Ann. 349 (2011), 459-508.

[3] C. Frohman and B. Fine, 'Some amalgam structures for Bianchi groups', Proc. Amer. Math. Soc. 102 (1988), 221-229.

[4] W. M. Goldman, Complex Hyperbolic Geometry (Oxford University Press, Oxford, 1999).

[5] D. D. Long, C. Maclachlan and A. W. Reid, 'Splitting groups of signature (1; n)', J. Algebra 185 (1996), 329-341.

[6] J.-P. Serre, Trees, Springer Monographs in Mathematics (Springer, Berlin, 1980).

[7] M. Stover, 'Property (FA) and lattices in SU(2, 1)', Internat. J. Algebra Comput. 17 (2007), 1335-1347.

JIEYAN WANG, College of Mathematics and Econometrics, Hunan University, Changsha, 410082, PR China e-mail: jywang@hnu.edu.cn

BAOHUA XIE, College of Mathematics and Econometrics, Hunan University, Changsha, 410082, PR China

e-mail: xiexbh@gmail.com 